\title{
Long-lived femtosecond photon echo in thin films on localized exciton states at room temperature
}

\author{
N.S. Vashurin ${ }^{1}$, V.O. Kompanets ${ }^{2}$, I.I. Popov ${ }^{1, *}$, S.E. Putilin ${ }^{3}$, and S.V. Chekalin ${ }^{2}$ \\ ${ }^{1}$ Volga State Technological University, 3 Lenin sq., Yoshkar-Ola, 424001 Russia \\ ${ }^{2}$ Institute of Spectroscopy of the Russian Academy of Sciences, 5 Fizicheskaya str, Moscow, Troitsk, \\ 108840 Russia \\ ${ }^{3}$ St. Petersburg National Research University of Information Technologies, Mechanics and Optics, \\ 49 Kronverksky Prospect, St. Petersburg, 197101 Russia
}

\begin{abstract}
Long-lived femtosecond photon echo was excited at room temperature in a two-photon regime on the charged exciton states (trion type) localized on the surface defects of a three-layer textured $\mathrm{ZnO} / \mathrm{Si}(\mathrm{B}) / \mathrm{Si}(\mathrm{P})$ film with the thickness of each layer at $100 \mathrm{~nm}$ in a longitudinal uniform magnetic field. The irreversible longitudinal relaxation time $T_{1}$ for the long-lived photon echo regime was of the order of $40 \mathrm{ps}$, whereas when measured with the help of the stimulated photon echo generated in the single-photon mode, the value was found not to exceed 600 fs.
\end{abstract}

The paper reports an experimental observation of the regime of a long-lived femtosecond photon echo at room temperature on exciton states localized on surface defects of a three-layer textured $\mathrm{ZnO} / \mathrm{Si}(\mathrm{B}) / \mathrm{Si}(\mathrm{P})$ with a thickness of each layer of $100 \mathrm{~nm}$. The excited film was exposed to a magnetic field applied in the direction that was longitudinal with respect to the propagation direction of the laser excitation. This textured film was obtained by magnetron sputtering. The decrease in the intensity of a femtosecond stimulated photon echo with increasing time interval between the second and third exciting impulses was recorded in the presence and absence of a uniform longitudinal magnetic field. The time interval between the first and second exciting pulses was chosen to be zero. The duration of the exciting impulses was of the order of $40 \mathrm{fs}$. The intensity of the applied magnetic field was $150 \mathrm{mT}$. The angle between the directions of propagation of the exciting pulses was about 9 degrees. The direction of recording the echo signal corresponded to the conditions of spatial synchronism for the stimulated photon echo excited in the two-photon regime.

In the presence of a longitudinal homogeneous magnetic field of the echo, a long-lived photon echo effect was observed in a three-layer film (Fig. 1), in single-layer films it did not. When an echo signal corresponding to the synchronism conditions for four-wave mixing and the stimulated photon echo excited in the single-photon mode was observed, the usual femtosecond stimulated photon echo (SPE) was recorded. The time of irreversible

\footnotetext{
* Corresponding author: Popov@,volgatech.net
} 
longitudinal relaxation $T_{1}$ for the regime of the long-lived photon echo was about $40 \mathrm{ps}$, whereas when it was measured with the help of the SPE generated in single-photon mode, a value of no more than $600 \mathrm{fs}$ was obtained.

The long-lived regime is realized, thanks to the presence of the spectral line, of trionic states formed not only in single-photon, but also in two-photon excitation regimes. Metastable (long-lived) quantum levels were excited in the two-photon regime.

The role of the magnetic field here has two functions. The first function, analogous to that shown by the authors of the work [1], is associated with periodic precession with the Larmor frequency of the magnetic moment of these two states, which ensures the transfer of the excitation of the usual quantum level to a long-lived one.

The second function of the magnetic field is associated with the rotation of the magnetic moment of the pseudo-electric dipoles arising during the formation of the photon echo around the direction of the longitudinally applied homogeneous magnetic field. In this case, the Larmor precession, acting on pseudoelectric dipoles, affects the phase precession rate of these dipoles. This contribution differs in sign for the upper (right-handed) and lower (lefthanded) components of the hyperfine structure of the excited level. Which ultimately leads to a non-Faraday rotation of the polarization vector of the echo signal.

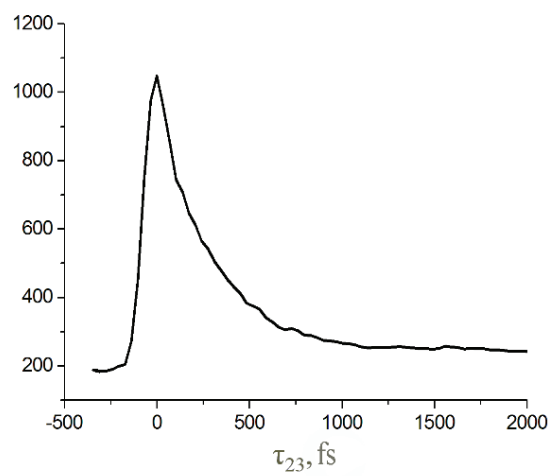

Fig. 1. Dependence of the intensity of the photon echo excited in the two-photon mode in the presence of a longitudinal homogeneous field on localized exciton states (such as the trion), on the value of the time interval between the second and third exciting impulses.

\section{References}

[1] L. Langer, S.V. Poltavtsev, I.A. Yugova et al., Phys. Rev. Lett. 109, 157403 (2012)

[2] L. Langer, S.V. Poltavtsev, I.A. Yugova et al., Nature Photon. 8, 851 (2014) 